\title{
On the Stable Size Distribution of Populations Reproducing by Fission into Two Unequal Parts
}

\author{
H. J. A. M. HEIJMANS \\ Centrum voor Wiskunde \& Informatica, Kruislaan 413, 1098 SJ Amsterdam, The Netherlands \\ Received 31 January 1984; revised 21 May 1984
}

\begin{abstract}
A nonlinear model describing the dynamics of a continuous culture of cells characterized by their size only, and reproducing by fission into two unequal parts, is formulated. It is assumed that cells grow proportionally to their size. Using techniques from dynamical systems theory, we establish results concerning the existence of a globally stable equilibrium.
\end{abstract}

\section{INTRODUCTION}

It is generally accepted by cell biologists that cell size is one of the most decisive parameters as far as the individual dynamic behavior of a cell is concerned. (See $[1,2,3,4,8,15,16,20,21]$.) In addition cell size is an attractive parameter because of the relative ease and precision with which it can be measured. During the past twenty years size distributions of cell populations have become of increasing interest, because the instrumentation for obtaining them has improved considerably. (See $[2,4,21]$.)

One of the main purposes of studying theoretical growth models of cell populations is to compare the analytical results with the experimental data in order to test the validity of the model-in other words, to derive information about the dynamics of the individual (growth, death, division) from the dynamics of the population as a whole. One of the main problems is to find a model which is general enough to give an acceptable description of the biological reality and which does not contain too many parameters. In this context our contribution must be seen as an attempt to describe some features of proliferating cell populations and to provide some additional insight in this complex subarea of structured population dynamics.

In this paper we consider a continuous culture (see [12]) of proliferating cells which are assumed to be characterized by their size alone. Here "size" 
can be replaced by any other quantity obeying a physical conservation law, for instance weight or protein content. We assume that the growth of an individual is proportional to its size (exponential growth). (See $[1,2,3,4$, 15].) In general this assumption is very restrictive. However, Anderson et al. [1-4] concluded from their measurements of mammalian cells in suspension cultures that the cell size growth rate is approximately proportional to cell size. The reason for restricting ourselves to the case of exponential growth becomes perfectly clear in Section 5. The idea is the following. If the growth of a cell is proportional to its size, the dynamics of the total biomass, represented by the population, is described by an ordinary differential equation. As a consequence the nonlinearity, which makes the model rather intractable in the most general case, can be computed a priori, i.e. without knowledge of the size distribution. Furthermore we assume that the ratio $P$ of the birth size of a daughter cell to the division size of her mother is a random variable described by a smooth probability density function, which does not depend on the division size of the mother and which is symmetric around $\frac{1}{2}$. This assumption was first suggested by Koch and Schaechter [15].

\section{THE MODEL}

We consider a population of cells contained in some completely stirred tank of volume $V$. The population is supplied at a constant rate $Q$ with fresh medium containing nutrients essential for their growth, and at the same rate medium containing cells and nutrient is removed from the tank. The ratio $D=Q / V$ is called the dilution rate, and is a control variable of the process. We assume that one main compound $S$ of the medium is needed to describe the dynamics of the cell population. This compound is called the growthlimiting substrate (or nutrient). In literature a population living in such environment is called a continuous culture. (See e.g. [12].)

We assume that the individuals of the cell population are characterized by their size $x$ only. (The state of an individual is its size; see [5].) An individual's size increases deterministically according to the ordinary differential equation

$$
\frac{d x}{d t}=g(x)
$$

$g$ is called the individual growth rate and, although this is not expressed in our notation, may also depend on environmental factors such as nutrient concentration. For some populations, $g(x)$ is found to be proportional to $x$ :

$$
g(x)=\gamma x,
$$


in which case we speak of exponential individual growth, because in this case (1.1) has the solution $x(t)=x(0) e^{\gamma t}$, if $\gamma$ does not depend on time $t$. In this paper we assume that the individual growth rate is given by (1.2) where $\gamma$ is not necessarily constant, but may depend on the substrate concentration $S$ in the tank.

Let the organisms reproduce by fission into two parts, and let the probability per unit of time that a cell of size $x$ divides be described by a function $b(x)$. The ratio $p$ of the size at birth of a daughter cell and the size at division of her mother is a random variable with smooth probability density function $d(p)$, which does not depend on the division size of the parent. Observe that $d(p)$ has to be symmetric around $p=\frac{1}{2}$. Notice that $\int_{0}^{1} d(p) d p=1$. (We refer to $[15,16,21]$ for more details.)

Our system can be described by two nonlinear equations

$$
\begin{aligned}
\frac{\partial n}{\partial t}(t, x)+ & \frac{\partial}{\partial x}[\gamma(S) x n(t, x)] \\
& =-D n(t, x)-b(x) n(t, x)+2 \int_{0}^{1} \frac{d(p)}{p} b\left(\frac{x}{p}\right) n\left(t, \frac{x}{p}\right) d p \\
& \frac{d S}{d t}=-\frac{1}{\theta} \gamma(S) \int_{0}^{\infty} x n(t, x) d x+D\left(S^{\text {in }}-S\right)
\end{aligned}
$$

where $n(t, x)$ is the (unknown) population density distribution, i.e., $\int_{x_{1}}^{x_{2}} n(t, x) d x$ is the number of cells with size between $x_{1}$ and $x_{2}$ at time $t$ per unit of volume. $S^{\text {in }}$ is the input nutrient concentration and $\theta$ is the so-called yield constant, i.e. the ratio (biomass of the organism formed)/(mass of substrate used). We assume that $\gamma(S)$ has the form of a hyperbola:

$$
\gamma(S)=\frac{m S}{k+S} .
$$

This was experimentally found by Monod. (See e.g. [12].) $m$ is called the maximum growth rate, and $k$ is the Michaelis-Menten constant. However, we wish to point out that this assumption is not essential for our calculations. The analysis can be carried through for more general $\gamma$.

The last two terms at the right-hand side of (1.3) describe the population's reproduction process. The factor $1 / p$ accounts for the fact that newborn cells with size in $(x, x+d x)$ come from a mother with size in the interval $(x / p, x / p+d x / p)$ which is $1 / p$ times as large. We do not give a derivation of Equation (1.3), but instead refer to a paper of Frederickson et al [8].

The first expression on the right-hand side of (1.4) accounts for the amount of substrate used by the individuals of the population for their 
growth (per unit of volume). Notice that

$$
W(t)=\int_{0}^{\infty} x n(t, x) d x
$$

is the biomass concentration. The second expression on the right-hand side of (1.4) is the difference of input and output of substrate.

From now on we assume that the following conditions are satisfied:

$\left(\mathrm{H}_{b}\right)(1) b(x)$ is continuous on $[0,1)$.

(2) $b(x)=0,0 \leqslant x \leqslant a, b(x)>0, a<x<1$.

(3) $\lim _{x \uparrow 1} \int_{a}^{x} b(\xi) d \xi=\infty$.

(4) $\frac{b(x)}{g(x)} \exp \left(-\int_{a}^{x} \frac{b(\xi)}{g(\xi)} d \xi\right)$ is bounded on $[a, 1]$.

$\left(\mathrm{H}_{d}\right)(1) d(p)=0$ outside $\left(\frac{1}{2}-\Delta, \frac{1}{2}+\Delta\right)$, where $0<\Delta<\frac{1}{2}$.

(2) $d$ is piecewise $C^{1}$ on $\left(\frac{1}{2}-\Delta, \frac{1}{2}+\Delta\right)$ with bounded derivative.

Condition $\left(\mathrm{H}_{b}\right)$ describes the following biological situation. Cells cannot divide before they reach a minimal size $a>0$, and they have to divide before they reach a maximal size, which is normalized to be 1 . The last assumption in $\left(\mathrm{H}_{b}\right)$ means that the function describing the chance per unit of size that a cell will divide at size $x$, remains bounded. (See Section 9.) In Equation (1.3) one must take $b(x / p) n(t, x / p)=0$ if $x / p>1$. We introduce the following notation:

$$
\alpha=\left(\frac{1}{2}-\Delta\right) a, \quad \beta=\frac{1}{2}+\Delta .
$$

$\alpha$ and $\beta$ can be interpreted respectively as the minimum and maximum size of a newborn cell. The fact that cells with size smaller than $\alpha$ cannot exist is expressed by the boundary condition

$$
n(t, \alpha)=0
$$

Furthermore we supplement (1.3)-(1.4) with the initial conditions

$$
\begin{gathered}
n(0, x)=n_{0}(x) \geqslant 0, \\
S(0)=S_{0}>0 .
\end{gathered}
$$

The first part of this paper (Sections 2,3,4) is concerned with the investigation of the corresponding linear equation in a slightly more general form. By means of an elementary transformation, it is reduced to a more tractable problem. This is done in Section 2. In Section 3 the associated eigenvalue problem is treated. Section 4 is concerned with the time-dependent linear equation. We prove that its solutions can be represented by a 
strongly continuous semigroup. The results of Section 3 are used to establish the large-time behavior of these solutions. In Section 5 it is explained how Equation (1.4) can be solved a priori, i.e. without knowledge of the solution of (1.3). Existence and uniqueness of solutions of the nonlinear problem (1.3)-(1.7) is proved in Section 6. In Section 7 we state our main result, which says that there exists a globally stable equilibrium. The proof of this result can be found in Section 8. In Section 9 we make some final remarks.

\section{TRANSFORMATION OF THE LINEAR EQUATION}

A good starting point for our investigation is the linear equation associated with (1.3). For the sake of generality (and because it causes no extra difficulties) we shall deal with a slightly more general form of this linear equation,

$$
\begin{aligned}
& \frac{\partial n}{\partial t}(t, x)+\frac{\partial}{\partial x}[g(x) n(t, x)]=-(D(x)+b(x)) n(t, x)
\end{aligned}
$$

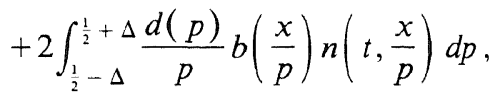

$$
\begin{aligned}
& n(t, \alpha)=0 .
\end{aligned}
$$

In words: we do not restrict ourselves to the case of exponential individual growth characterized by (1.2), and the death rate $D$ (= dilution rate if it concerns a continuous culture) is allowed to depend on $x$. We make the following assumptions on $g$ and $D$.

$\left(\mathrm{H}_{g}\right) \mathrm{g}$ is a strictly positive, continuous function on $[\alpha, 1]$.

$\left(\mathrm{H}_{D}\right) \quad D$ is a nonnegative, integrable function on $[\alpha, 1]$.

Now let us define

$$
E(x)=\exp \left(-\int_{\alpha}^{x} \frac{b(\xi)+D(\xi)}{g(\xi)} d \xi\right)
$$

This quantity has a clear biological interpretation. From a cohort of $N$ individuals starting at size $\alpha, N \cdot E(x)$ will reach $x$ without having died (been washed out) or divided. Observe that $E(1)=0$. Equation (2.1) is supplemented with the initial condition

$$
n(0, x)=n_{0}(x)
$$

It is suggested by the biological interpretation of $n(t, x)$ that for all $t \geqslant 0$, 
both $n(t, \cdot)$ and $b(\cdot) n(t, \cdot)$ should be integrable. We expect that, if similar conditions are imposed on the initial function $n_{0}(\cdot)$, together with $n_{0}(x) \geqslant 0$, a.e. on $[\alpha, 1]$, then $(2.1)$ has a solution also satisfying these conditions. However, guided by the desire for mathematical simplicity we shall impose a more restrictive condition on $n_{0}$ :

$\left(\mathrm{H}_{n_{0}}\right) \frac{n_{0}(x)}{E(x)}$ is integrable on $[\alpha, 1]$.

It is implied by the results of section 4 that this property is inherited by the solutions $n(t, \cdot)$ of (2.1)-(2.3). With this in mind, the following transformation does not come out of the blue:

$$
m(t, x)=\frac{g(x)}{E(x)} n(t, x) .
$$

We obtain the following initial-value problem for $m(t, x)$ :

$$
\begin{gathered}
\frac{\partial m}{\partial t}+g(x) \frac{\partial m}{\partial x}=\int_{\frac{1}{2}-\Delta}^{\frac{1}{2}+\Delta} k(p, x) m\left(t, \frac{x}{p}\right) d p, \\
m(t, \alpha)=0, \\
m(0, x)=\phi(x):=\frac{g(x)}{E(x)} n_{0}(x),
\end{gathered}
$$

where

$$
k(p, x)=\frac{2}{p} d(p) b(x / p) \frac{g(x)}{g(x / p)} \frac{E(x / p)}{E(x)} .
$$

One should take $k(p, x) m(t, x / p)=0$ if $x / p>1$.

For all $p \in\left[\frac{1}{2}-\Delta, \frac{1}{2}+\Delta\right]$, the function $k(p, \cdot)$ has support $[p a, p)$ (which is contained in $[\alpha, \beta)$ ) and is bounded because of assumption $\left(\mathrm{H}_{b}\right)(4)$.

Condition $\left(\mathrm{H}_{n_{0}}\right)$ yields that $\phi$ is an integrable function, i.e. $\phi \in L_{1}[\alpha, 1]$. Most of the time we shall write $L_{1}$ if we mean $L_{1}[\alpha, 1]$. We shall look for solutions $m(t, x)$ of $(2.5)-(2.7)$ satisfying $m(t, \cdot) \in L_{1}$, for all $t \geqslant 0$.

Equations (2.5)-(2.7) can be rewritten as an abstract Cauchy problem.

$$
\begin{aligned}
\frac{d m}{d t} & =A m, \quad t>0, \\
m(0) & =\phi, \quad \phi \in L_{1},
\end{aligned}
$$

where $A$ is the closed operator on $L_{1}$ given by

$$
(A \psi)(x)=-g(x) \frac{d \psi}{d x}+\int_{\frac{1}{2}-\Delta}^{\frac{1}{2}+\Delta} k(p, x) \psi\left(\frac{x}{p}\right) d p
$$


for all $\psi$ in the domain $\mathbf{D}(A)$ of $A$ :

$$
\mathrm{D}(A)=\left\{\psi \in L_{1} \mid \psi \text { is absolutely continuous and } \psi(\alpha)=0\right\}
$$

Finally, we refer to [6], where Diekmann et al. discuss a similar problem and use the same transformation (2.4). However, they work in the space of continuous functions, whereas we work in $L_{1}$. The reason for this becomes clear in Lemma 8.1, where we prove boundedness of solutions of the nonlinear problem.

\section{THE LINEAR EIGENVALUE PROBLEM}

This section is entirely concerned with the investigation of the spectrum $\sigma(A)$ of the operator $A$ given by (2.11)-(2.12). The results that we shall find can be used to characterize the behavior of the solutions of the time-dependent linear equation (2.1)-(2.3) for $t \rightarrow \infty$.

Throughout this paper we use the following notation. For an operator $L$ we denote by $\operatorname{P\sigma }(L)$ the point spectrum of $L . \mathbf{N}(L)$ is the nullspace of $L$, and $\mathbf{R}(L)$ the range.

We are looking for sclutions $\psi \in \mathbf{D}(A)$ of the inhomogeneous equation

$$
\lambda \psi-A \psi=f,
$$

where $f \in L_{1}$. Let

$$
G(x)=\int_{\alpha}^{x} \frac{d \xi}{g(\xi)}, \quad \alpha \leqslant x \leqslant 1
$$

and

$$
\phi(x)=e^{\lambda G(x)} \psi(x) .
$$

Substitution of (3.3) in (3.1) using (2.11) yields

$$
\frac{d \phi}{d x}=\int_{\frac{1}{2}-\Delta}^{\frac{1}{2}+\Delta} \hat{k}_{\lambda}(p, x) \phi\left(\frac{x}{p}\right) d p+f(x) e^{\lambda G(x)},
$$

where

$$
\hat{k}_{\lambda}(p, x)=\frac{k(p, x)}{g(x)} e^{-\lambda[G(x / p)-G(x)]} .
$$

Integration from $\alpha$ to $x$ and using that $\phi(\alpha)=0$ [because $\psi \in \mathbf{D}(A)$ ] yields

$$
\begin{aligned}
\phi(x)= & \int_{\frac{1}{2}-\Delta}^{\frac{1}{2}+\Delta}\left(\int_{\alpha}^{(x, \beta)^{-}} \hat{k}_{\lambda}(p, \xi) \phi(\xi / p) d \xi\right) d p \\
& +\int_{\alpha}^{x} f(\xi) e^{\lambda G(\xi)} d \xi
\end{aligned}
$$


where $(x, \beta)^{-}$stands for the minimum of $x$ and $\beta$. We can write (3.5) abstractly as

$$
\phi=T_{\lambda} \phi+U_{\lambda} f
$$

where $T_{\lambda}$ and $U_{\lambda}$ are given by

$$
\begin{aligned}
& \left(T_{\lambda} \psi\right)(x)=\int_{\frac{1}{2}-\Delta}^{\frac{1}{2}+\Delta}\left(\int_{\alpha}^{(x, \beta)^{-}} \hat{k}_{\lambda}(p, \xi) \psi(\xi / p) d \xi\right) d p \\
& \left(U_{\lambda} \psi\right)(x)=\int_{\alpha}^{x} \psi(\xi) e^{\lambda G(\xi)} d \xi
\end{aligned}
$$

for all $\psi \in L_{1}$. Obviously $T_{\lambda}$ and $U_{\lambda}$ define bounded, linear operators from $L_{1}$ into $L_{1}$.

The following result is straightforward.

THEOREM 3.1

Let $f \in L_{1}$. Then $\psi \in \mathbf{D}(A)$ is a solution of the inhomogeneous equation (3.1) if and only if $\phi$ given by (3.3) is a solution of (3.6).

The advantage of this reformulation should become clear to the reader when we state our next result.

THEOREM 3.2

$T_{\lambda}$ and $U_{\lambda}$ define completely continuous operators.

The proof of this result is evident and will be omitted. The sense of the following definition should be clear from Theorem 3.1:

$$
\Sigma=\left\{\lambda \in C \mid 1 \in P \sigma\left(T_{\lambda}\right)\right\} .
$$

Notice that $\sigma\left(T_{\lambda}\right) \backslash\{0\}=P \sigma\left(T_{\lambda}\right) \backslash\{0\}$ because $T_{\lambda}$ is completely continuous. THEOREM 3.3

$\sigma(A)=P \sigma(A)=\Sigma$. If $\lambda \notin \sigma(A)$, then the resolvent of $A, R_{\lambda}(A)=(\lambda I-$ $A)^{-1}$, is completely continuous.

Proof. It is obvious from Theorem 3.1 that $\Sigma=P \sigma(A)$. Now suppose that $\lambda \notin P \sigma(A)$. Consequently $1 \notin \sigma\left(T_{\lambda}\right)$, and we conclude that $\phi-T_{\lambda} \phi=$ $U_{\lambda} f$ is solvable for all $f \in L_{1}$. From Theorem 3.1 we conclude that $\lambda \notin \sigma(A)$. For $\lambda \notin \sigma(A)$ the resolvent is given by $\left(R_{\lambda}(A) f\right)(x)=e^{-\lambda G(x)}[(I-$ $\left.\left.T_{\lambda}\right)^{-1} U_{\lambda} f\right](x)$, and from Theorem 3.2 we deduce that $R_{\lambda}(A)$ is completely continuous.

Most results in the remainder of this section will be given without proof. Instead, we refer to another paper of ours [11], where a similar eigenvalue 
problem has been treated, and the reader will have no difficulty in seeing that many results of that paper can be carried over to our present case.

THEOREM 3.4

$\boldsymbol{\sigma}(A)$ consists of isolated eigenvalues.

Proof. See [11, Theorem 2.4].

An important quantity is the dominant eigenvalue of $A$, i.e. the eigenvalue with largest real part. (In practical cases the dominant eigenvalue happens to be real.) In many cases positive-operator theory can be used to prove the existence of a dominant eigenvalue.

Let the positive cone $L_{1}^{+}$be defined by

$$
L_{1}^{+}=\left\{\psi \in L_{1} \mid \psi(x) \geqslant 0 \text { a.e. on }[\alpha, 1]\right\} \text {. }
$$

The dual cone of $L_{1}^{+}$is $L_{\infty}^{+}$, i.e. the subset of functions in $L_{\infty}[\alpha, 1]$ which are nonnegative a.e. For $\lambda \in R$ we have that $T_{\lambda}$ is positive with respect to the cone $L_{1}^{+}$. For the basic theory concerning positive cones and positive operators we refer to the monograph of Schaefer [19].

In [18] Sawashima introduced the very useful notion of a nonsupport operator (which is more restrictive than just positivity).

DEFINITION [18]

Let $X^{+}$be a positive cone in the Banach space $X$, and let $\left(X^{+}\right)^{\star}$ be the dual cone. A positive operator $T: X \rightarrow X$ is called nonsupport with respect to $X^{+}$if for all $\psi \in X^{+}, \psi \neq 0$ and $F \in\left(X^{+}\right)^{\star}, F \neq 0$, there exists an integer $p$ such that for all $n \geqslant p$ we have $F\left[T^{n} \psi\right]>0$.

It can be shown with very little effort that $T_{\lambda}$ is nonsupport with respect to $L_{1}^{+}$for all $\lambda \in R$. As a matter of fact we have: There exists an integer $p$ such that for all $\psi \in L_{1}^{+}, \psi \neq 0$ and for all $x \in(\alpha, 1],\left(T_{\lambda}^{p} \psi\right)(x)>0$. (See also [11, Theorem 5.1].)

From this it follows that there exists a $\phi_{\lambda} \in L_{1}^{+}$and $F_{\lambda} \in L_{\infty}^{+}$such that

$$
\begin{aligned}
T_{\lambda} \phi_{\lambda} & =r_{\lambda} \phi_{\lambda}, \\
T_{\lambda}^{\star} F_{\lambda} & =r_{\lambda} F_{\lambda},
\end{aligned}
$$

where $r_{\lambda}=r\left(T_{\lambda}\right)$ is the spectral radius of $T_{\lambda}$ and $T_{\lambda}^{\star}: L_{\infty} \rightarrow L_{\infty}$ is the adjoint of $T_{\lambda}$. (See [18].) If $r\left(T_{\lambda}\right)=1$ then $\lambda \in \operatorname{P\sigma }(A)$. The equation $r\left(T_{\lambda}\right)$ $=1$ has a unique solution $\lambda_{d} \in R$ (See [11, Theorem 4.4]).

Now let

$$
\psi_{d}(x)=e^{-\lambda_{d} G(x)} \phi_{\lambda_{d}}(x),
$$


where $\phi_{\lambda_{d}}$ is determined by (3.11a). As in [11, Theorems 5.4, 6.2] it can be proved that the eigenvalue $\lambda_{d}$ of $A$ is algebraically simple and strictly dominant, i.e. if $\lambda \in \sigma(A), \lambda \neq \lambda_{d}$, then $\operatorname{Re} \lambda<\lambda_{d}$. We summarize some of our results.

\section{COROLLARY 3.5}

The operator $A$ has a dominant eigenvalue $\lambda_{d}$ which is algebraically simple. The corresponding eigenvector $\psi_{d}$ and adjoint eigenvector $F_{d}$ are positive. If $\lambda \in \operatorname{P\sigma }(A)$ and $\lambda \neq \lambda_{d}$, then $\operatorname{Re} \lambda<\lambda_{d}$.

Remark 3.1. The element $F_{d} \in L_{\infty}^{+}$can be computed from $F_{\lambda_{d}}$ given by (3.11b). (See [11, Section 7].) However, they do not coincide.

Remark 3.2. $\psi_{d}$ is absolutely continuous, because $\psi_{d} \in \mathbf{D}(A)$.

Remark 3.3. The fact that all eigenvectors of $A$ are (absolutely) continuous would have permitted us to work in the space of continuous functions instead of $L_{1}$. This is done in [11]. The results, however, remain the same.

As in $[11$, Section 6] we can compute the characteristic equation (i.e. the equation from which all eigenvalues of $A$ can be computed) for our problem. Let $e_{j} \in L_{1}, j \geqslant 1$, be given by the recurrent relation

$$
\begin{aligned}
& e_{1}(x) \equiv 1, \quad \alpha \leqslant x \leqslant 1, \\
& e_{j}(x)=-\int_{\frac{1}{2}-\Delta}^{\frac{1}{2}+\Delta}\left(\int_{(x, \beta)^{-}}^{\beta} \hat{k}_{\lambda}(p, \xi) e_{j-1}(\xi / p) d \xi\right) d p .
\end{aligned}
$$

Remark 3.4. $e_{j}$ does depend on $\lambda, j \geqslant 2$.

Let $q$ be the smallest integer such that $\alpha \geqslant\left(\frac{1}{2}+\Delta\right)^{q}$. Then the characteristic equation is given by

$$
\pi(\lambda):=\int_{\frac{1}{2}-\Delta}^{\frac{1}{2}+\Delta}\left(\int_{\alpha}^{\beta} \hat{k}_{\lambda}(p, \xi)\left\{e_{1}\left(\frac{\xi}{p}\right)+\cdots+e_{q-1}\left(\frac{\xi}{p}\right)\right\} d \xi\right) d p=1
$$

(see [11, Section 6]).

THEOREM 3.6

$\lambda \in \operatorname{Po}(A)$ if and only if $\pi(\lambda)=1$. In every finite vertical strip $\{\lambda \mid s \leqslant \operatorname{Re} \lambda$ $\leqslant t\}$ where $-\infty<s<t<\infty$, there are at most finitely many elements of $\sigma(A)$.

Proof. See [11, Theorems 6.5, 6.6].

Using the algebraic simplicity of the eigenvalue $\lambda_{d}$ and the compactness of the resolvent (cf. Theorem 3.3), we can give the following decomposition 
of the space $L_{1}$ :

$$
L_{1}=\mathbf{N}\left(\lambda_{d} I-A\right) \oplus \mathbb{R}\left(\lambda_{d} I-A\right) .
$$

Let $P$ be the projection on $\mathbf{N}\left(\lambda_{d} I-A\right)$ associated with this decomposition; then $P$ is given by

$$
P \phi=F_{d}[\phi] \psi_{d}, \quad \phi \in L_{1}
$$

where the linear functional $F_{d}$ is given by Corollary 3.5, and satisfies

$$
F_{d}\left[\psi_{d}\right]=1
$$

For our purposes the case of exponential individual growth and constant death rate (washout) is of special interest. Let $g(x)=\gamma x$ and $D(x)=D$. If $\alpha \geqslant\left(\frac{1}{2}+\Delta\right)^{2}$, then $\pi(\lambda)$ is given by

$$
\begin{aligned}
\pi(\lambda) & =\int_{\frac{1}{2}-\Delta}^{\frac{1}{2}+\Delta}\left(\int_{\alpha}^{\beta} \hat{k}_{\lambda}(p, \xi) d \xi\right) d p \\
& =2 \int_{\frac{1}{2}-\Delta}^{\frac{1}{2}+\Delta} d(p) \cdot p^{(\lambda+D) / \gamma} d p
\end{aligned}
$$

where we have used (2.8) and (3.4). Because of the symmetry of $d(p)$ around $p=\frac{1}{2}$ and the fact that $\int_{\frac{1}{2}-\Delta}^{\frac{1}{2}+\Delta} d(p) d p=1$ (which is clear from the interpretation) we have $2 \int_{\frac{1}{1}}^{\frac{1}{2}+\Delta} p d(p) d p=1$. Consequently the dominant eigenvalue $\lambda_{d}$ is determined by $\left(\lambda_{d}+D\right) / \gamma=1$; hence $\lambda_{d}=\gamma-D$. This result remains valid if $\alpha<\left(\frac{1}{2}+\Delta\right)^{2}$. To prove this, we consider the eigenvalue problem associated with the original equation (2.1).

$$
\begin{aligned}
\lambda n(x)+\frac{d}{d x}[\gamma x n(x)]= & -D n(x)-b(x) n(x) \\
& +2 \int_{\frac{1}{2}-\Delta}^{1}+\Delta \frac{d(p)}{p} b\left(\frac{x}{p}\right) n\left(\frac{x}{p}\right) d p .
\end{aligned}
$$

After multiplication with $x$ and integration from $\alpha$ to 1 we arrive at

$$
\lambda W-\gamma W=-D W
$$

where

$$
W=W[n]=\int_{\alpha}^{1} x n(x) d x
$$

can be interpreted as the biomass associated with the size distribution $n$. The 
eigenvector $n_{d}$ associated with the dominant eigenvalue $\lambda_{d}$ is given by

$$
n_{d}(x)=\frac{E(x)}{\gamma x} \psi_{d}(x),
$$

where $\psi_{d}$ is given by (3.12). So $n_{d}(x)$ is positive a.e., and as a consequence $W\left[n_{d}\right]>0$, from which we conclude that $\lambda_{d}=\gamma-D$.

Let $F_{d}$ be the associated adjoint eigenvector (see Corollary 3.5). We shall prove that $F_{d}=F$, where $F$ is given by

$$
F[\phi]=\int_{\alpha}^{1} \phi(x) E(x) d x, \quad \phi \in L_{1},
$$

by showing that for this $F$ we have

$$
F[A \psi]=(\gamma-D) F[\psi] \quad \text { for all } \psi \in \mathbb{D}(A) .
$$

Now let $\psi \in \mathbf{D}(A)$. We have

$$
\begin{aligned}
F[A \psi]= & \int_{\alpha}^{1}\left\{-\gamma x \frac{d \psi}{d x}+\int_{\frac{1}{2}-\Delta}^{1}+\Delta k(p, x) \psi\left(\frac{x}{p}\right) d p\right\} E(x) d x \\
= & \int_{\alpha}^{1} \psi(x) \frac{d}{d x}(\gamma x E(x)) d x \\
& +\int_{\alpha}^{1}\left\{2 \int_{\frac{1}{2}-\Delta}^{1}+\Delta d(p) b\left(\frac{x}{p}\right) E\left(\frac{x}{p}\right) \psi\left(\frac{x}{p}\right) d p\right\} d x \\
= & (\gamma-D) \int_{\alpha}^{1} \psi(x) E(x) d x-\int_{\alpha}^{1} b(x) E(x) \psi(x) d x \\
& +2 \int_{\frac{1}{2}-\Delta}^{\frac{1}{2}+\Delta} p d(p) d p \cdot \int_{\alpha}^{1} b(\xi) E(\xi) \psi(\xi) d \xi \\
= & (\gamma-D) \int_{\alpha}^{1} \psi(x) E(x) d x=(\gamma-D) F[\psi],
\end{aligned}
$$

where we have used that $2 \int_{\frac{1}{2}}^{\frac{1}{2}+\Delta} p d(p) d p=1$.

We summarize our results in the following theorem.

THEOREM 3.7

If $g(x)=\gamma x$ and $D(x)=D$ for all $x \in[\alpha, 1]$, then the dominant eigenvalue $\lambda_{d}$ of $A$ is given by $\lambda_{d}=\gamma-D$. The associated adjoint eigenvector $F_{d}$ is given by $F_{d}[\phi]=\int_{\alpha}^{1} \phi(x) E(x) d x$.

\section{THE LINEAR TIME-DEPENDENT PROBLEM}

In this section we shall investigate the initial-value problem (2.5)-(2.7), and we shall prove the existence and uniqueness of solutions and determine 
their behavior for large $t$. All the results in this section are based on semigroup methods. Readers unfamiliar with the theory of semigroups are referred to $[17,22]$.

In Section 2 we have rewritten (2.5)-(2.7) as the abstract Cauchy problem (2.9)-(2.10). We write

$$
A=B+C,
$$

where

$$
\begin{aligned}
& (B \psi)(x)=-g(x) \frac{d \psi}{d x}, \\
& (C \psi)(x)=\int_{\frac{1}{2}-\Delta}^{\frac{1}{2}+\Delta} k(p, x) \psi\left(\frac{x}{p}\right) d p
\end{aligned}
$$

$B$ is an unbounded closed operator on $L_{1}$ with domain

$$
\mathbf{D}(B)=\left\{\psi \in L_{1} \mid \psi \text { is absolutely continuous and } \psi(\alpha)=0\right\} \text {, }
$$

and $C$ defines a bounded operator. With little effort one can see that $B$ generates a strongly continuous semigroup $e^{t B}$ given by

$$
\left(e^{t B} \psi\right)(x)=\psi\left(G^{-1}(G(x)-t)\right), \quad t \geqslant 0,
$$

where $G^{-1}$ denotes the inverse of the function $G$ given by (3.2). One should read $G^{-1}(\tau)=0$ if $\tau<0$. Obviously

$$
e^{t B}=0 \quad \text { if } \quad t \geqslant G(1)
$$

Now a standard result from semigroup theory (see [17, Chapter 3, Theorem 1.1]) yields that $A=B+C$ generates a strongly continuous semigroup as well, because $C$ is bounded. We denote this semigroup by $T(t)$.

\section{THEOREM 4.1}

A generates a strongly continuous semigroup $T(t)$.

This proves the existence and uniqueness of solutions of the initial value problem (2.5)-(2.7). If we denote this solution by $m(t, \cdot ; \phi)$ or also $m(t ; \phi)$, then $m(t ; \phi)=T(t) \phi$.

Remark 4.1. $m(t ; \phi)=T(t) \phi$ is not a solution of $(2.5)-(2.7)$ in the strong sense of the word. More precisely, $m(t, x ; \phi)$ is not necessarily differentiable with respect to $t$ and $x$ separately. [This is only true if $\phi \in \mathbf{D}(A)$.] It can be shown however, that $m(t, x ; \phi)$ is differentiable along 
the characteristics $t-G(x)=$ const of the PDE given by (2.5):

$$
\lim _{h \rightarrow 0} \frac{m\left(t+h, G^{-1}(G(x)+h)\right)-m(t, x)}{h}=\int_{\frac{1}{2}-\Delta}^{\frac{1}{2}+\Delta} k(p, x) m\left(t, \frac{x}{p}\right) d p,
$$

where $m(t, x)=m(t, x ; \phi)$.

It is possible to obtain the solutions $m(t ; \phi)$ explicitly as a series. Applying the variation-of-constants formula, the abstract Cauchy problem (2.9)-(2.10) leads to the integral equation

$$
m(t ; \phi)=e^{t B} \phi+\int_{0}^{t} e^{(t-s) B} C m(s ; \phi) d s .
$$

Let

$$
\begin{aligned}
m_{0}(t ; \phi) & =e^{t B} \phi \\
m_{i+1}(t ; \phi) & =\int_{0}^{t} e^{(t-s) B} C m_{i}(s ; \phi) d s, \quad i \geqslant 0 ;
\end{aligned}
$$

then the solution $m(t ; \phi)$ is given by

$$
m(t ; \phi)=\sum_{i=0}^{\infty} m_{i}(t ; \phi) .
$$

This series representation of the solution has a clear biological interpretation, $m_{0}(t ; \phi)$ represents the 0 th generation at time $t$, i.e. all individuals which were present at time $t=0$ and have not yet died or divided. Inductively the $i$ th generation $m_{i}(t ; \phi)$ contains all daughters of cells of the $(i-1)$ th generation.

For the proof of the following result we refer to [6, Lemma 4.1]. THEOREM 4.2

At every (finite) time instant $t$ only a finite number of generations are present in the population.

The asymptotic behavior of the solutions for large $t$ can be determined relatively easily if one is able to prove compactness of the semigroup after finite time. Compactness of a semigroup means, among other things, that the initial function is smoothed if the semigroup acts on it.

THEOREM 4.3

$T(t)$ is compact for $t \geqslant G(1)$.

Observe that $G(1)$ is the time instant at which the 0th generation goes extinct. [See (4.5).] Theorem 4.3 is proved in the Appendix for the case 
$g(x)=\gamma x$. We restrict ourselves to this situation for several reasons. First of all, this is exactly the case for which the compactness property is not fulfilled if fission occurs into two equal parts [i.e. $d(p)=\delta\left(p-\frac{1}{2}\right)$ ]. (see [6, Section 8] and Remark 4.2 below.) Secondly, this happens to be the case that we are mainly interested in. However, it can be checked rather easily that the result remains valid for all functions $g$ satisfying $\left(\mathrm{H}_{g}\right)$.

Remark 4.2. In [6] the dynamics of a population reproducing by fission into two equal parts is investigated rigorously. In that case the semigroup is compact after finite time if some condition on the growth rate $g(x)$ is fulfilled. It is proved among other things that:

(1) If $g(2 x)<2 g(x)$ for all $x$ [or $g(2 x)>2 g(x)$ ], then $T(t)$ is compact after finite time.

(2) $T(t)$ never becomes compact if $g(2 x)=2 g(x)$ for all $x \cdot[g(x)=\gamma x$ is an important example of this situation.]

Biologically the relation $g(2 x)=2 g(x)$ means that the size of the offspring of some mother does not depend on the moment of fission of that mother, so that the property "equal size" is hereditary. This, of course, is not true if a cell can divide into two unequal parts.

Now let $\psi_{d}$ be the eigenvector of $A$ associated with the dominant eigenvector $\lambda_{d}$ of $A$ (cf. Corollary 3.5). Then

$$
T(t) \psi_{d}=e^{\lambda_{d} t} \psi_{d}
$$

describing the action of $T(t)$ on $\mathbf{N}\left(\lambda_{d} I-A\right)$. For the action of $T(t)$ on $\mathbf{R}\left(\lambda_{d} I-A\right)=\mathbf{N}(P)=\mathbf{R}(I-P)$, where $P$ is the projection given by (3.15), we can deduce an exponential estimate.

LEMMA 4.4

There exist positive constants $\epsilon$ and $K$ such that for all $\phi \in L_{1}$

$$
\|(I-P) T(t) \phi\| \leqslant K e^{\left(\lambda_{d}-\epsilon\right) t}\|\phi\| .
$$

Proof. Theorem 3.6 yields that there exists a constant $\epsilon>0$ such that for all $\lambda \in \sigma(A), \lambda \neq \lambda_{d}$ we have $\operatorname{Re} \lambda \leqslant \lambda_{d}-\epsilon$. From Theorem 4.3 we conclude that

$$
\sigma(T(t)) \backslash\{0\}=P \sigma(T(t)) \backslash\{0\}=\left\{e^{\lambda t} \mid \lambda \in \sigma(A)\right\} .
$$

(See [17, Chapter 2, Section 2.2].) Let $\hat{A}$ denote the restriction of $A$ to $\mathbf{R}\left(\lambda_{d} I-A\right)$; then $\sigma(\hat{A})=P \sigma(\hat{A})=P \sigma(A) \backslash\left\{\lambda_{d}\right\} \subset\left\{\lambda \mid \operatorname{Re} \lambda \leqslant \lambda_{d}-\epsilon\right\}$. A result of Hale $[10, \S 7.4]$ completes the proof. 
Combination of this lemma and (4.10) yields the following result. COROLLARY 4.5

For all $\phi \in L_{1}$ we have

$$
\left\|T(t) \phi-F_{d}[\phi] e^{\lambda_{d} t} \psi_{d}\right\| \leqslant K e^{\left(\lambda_{d}-\epsilon\right) t}\|\phi\|
$$

where $F_{d}$ is normalized by the condition (3.16).

If $\phi \geqslant 0, \phi \neq 0$, then $F_{d}[\phi]>0$. A similar result can be stated in terms of the original problem (2.1)-(2.3). We shall do this for the case $g(x)=\gamma x$, $D(x)=D, x \in[\alpha, 1]$, which is of special interest to us. Let $n_{0}$ satisfy condition $\left(\mathrm{H}_{n_{0}}\right)$. By $n\left(t ; n_{0}\right)$ we denote the solution of (2.1)-(2.3). Let $\phi$ be given by (2.7), i.e. $\phi(x)=[\gamma x / E(x)] n_{0}(x)$. Theorem 3.7 yields that

$$
F_{d}[\phi]=\int_{\alpha}^{1} \phi(x) E(x) d x=\int_{\alpha}^{1} \gamma x n_{0}(x) d x=\gamma W\left[n_{0}\right],
$$

where $W$ is given by (3.16). Let $n_{d}$, given by (3.17), be normalized by the condition

$$
W\left[n_{d}\right]=1
$$

[See (3.16).]

COROLLARY 4.6

Let $g(x)=\gamma x$ and $D(x)=D$, for all $x \in[\alpha, 1]$. Let $n_{0} \in L_{1}$ satisfy $\left(\mathrm{H}_{n_{0}}\right)$. Then $\left\|n\left(t ; n_{0}\right)-W\left[n_{0}\right] e^{\lambda_{d} t} n_{d}\right\|<M e^{\left(\lambda_{d}-\epsilon\right) t}\left\|n_{0}\right\|$, where $M$ is a positive constant not depending on $n_{0}$.

\section{THE DYNAMICS OF SUBSTRATE AND BIOMASS}

An important feature of the nonlinear model discussed in Section 1, which also explains why we restrict ourselves to the case of exponential individual growth, is the following. If we multiply (1.3) on both sides by $x$ and integrate over all sizes $x$, we find a balance equation describing the evolution of the biomass concentration. This equation turns out to be an ordinary differential equation:

$$
\frac{d W}{d t}=(\gamma(S)-D) W
$$

Equation (1.4) describes the evolution of the substrate concentration, and for convenience we write it down once more:

$$
\frac{d S}{d t}=-\frac{1}{\theta} \gamma(S) W+D\left(S^{\text {in }}-S\right) .
$$


These equations are to be supplemented with the initial conditions

$$
\begin{aligned}
& W(0)=w, \\
& S(0)=S_{0},
\end{aligned}
$$

where $w$ is the biomass represented by the initial size distribution $n_{0}$,

$$
w=W\left[n_{0}\right]=\int_{\alpha}^{1} x n_{0}(x) d x,
$$

and $S_{0}>0$ is some fixed quantity. Summarizing, we might say that the evolution of biomass and substrate concentration in the tank reactor is described by a system of two ordinary differential equations. Mathematically this means that the nonlinearity $\gamma(S)$ can be computed a priori, i.e. without knowledge of the solution. The situation turns out to be much more complicated if growth of an individual is not proportional to its size.

Equations (5.1) and (5.2) happen to be the equations originally found by Monod (see e.g. [12]). They have been extensively investigated by Hsu et al. [13] (who deal with the more general situation that several species are competing for nutrients), and for the following we refer to their paper.

We denote the solutions of (5.1)-(5.4) by $W\left(t ; S_{0}, w\right)$ and $S\left(t ; S_{0}, w\right)$. THEOREM 5.1 [13]

The solutions $W\left(t ; S_{0}, w\right)$ and $S\left(t ; S_{0}, w\right)$ of (5.1)-(5.4) are positive and bounded.

The system (5.1)-(5.2) always has the trivial equilibrium

$$
W=0, \quad S=S^{\text {in }}
$$

There exists a nontrivial equilibrium

$$
\begin{aligned}
& W=W_{e}=\theta\left(S^{\text {in }}-\frac{k D}{m-D}\right), \\
& S=S_{e}=\frac{k D}{m-D}
\end{aligned}
$$

if and only if the following conditions are satisfied:

$$
\left(\mathrm{H}_{e}\right) m>D \text { and } \frac{k D}{m-D}<S^{\mathrm{in}} \text {. }
$$

THEOREM 5.2 [13]

Let $S_{0}>0$ and $w>0$. If $\left(\mathrm{H}_{e}\right)$ is not satisfied, then

$$
\lim _{t \rightarrow \infty} W\left(t ; S_{0}, w\right)=0, \quad \lim _{t \rightarrow \infty} S\left(t ; S_{0}, w\right)=S^{\text {in }} .
$$


If $\left(\mathrm{H}_{e}\right)$ is satisfied, then

$$
\lim _{t \rightarrow \infty} W\left(t ; S_{0}, w\right)=W_{e}, \quad \lim _{t \rightarrow \infty} S\left(t ; S_{0}, w\right)=S_{e} .
$$

Remark 5.1. $Z(t)$ defined by $Z(t)=S\left(t ; S_{0}, w\right)+(1 / \theta) W\left(t ; S_{0}, w\right)$ obeys the ODE $d Z / d t=D\left(S^{\text {in }}-Z\right)$, having the general solution $Z(t)=S^{\text {in }}$ $+C e^{-D t}$, where $C$ is a constant.

Summarizing, we might say that biomass and substrate concentration tend to a globally stable equilibrium if $t$ becomes large. Our main question is whether a similar result holds for the size distribution. Before answering this question we have to deal with the problem of the existence and uniqueness of solutions.

\section{EXISTENCE AND UNIQUENESS OF SOLUTIONS}

In the preceding section we observed that the nonlinearity $\gamma(S)$ can be computed a priori. Let

$$
\gamma\left(t ; S_{0}, w\right)=\gamma\left(S\left(t ; S_{0}, w\right)\right) .
$$

If there is no likely confusion we shall write $\gamma(t)$ instead of $\gamma\left(t ; S_{0}, w\right)$. We introduce the new time variable $\tau$, given by

$$
\tau=\tau(t)=\int_{0}^{t} \gamma(s) d s,
$$

and we denote its inverse by $t=t(\tau)$. Let $u(\tau, x)$ be given by

$$
e^{D t} n(t, x)=u(\tau, x) ;
$$

then $u(\tau, x)$ obeys the equation

$$
\begin{aligned}
\frac{\partial u}{\partial t}+\frac{\partial}{\partial x}[x u(\tau, x)]= & -\frac{b(x)}{\hat{\gamma}(\tau)} u(\tau, x) \\
& +2 \int_{\frac{1}{2}-\Delta}^{\frac{1}{2}+\Delta} \frac{d(p)}{p} \frac{b(x / p)}{\hat{\gamma}(\tau)} u\left(\tau, \frac{x}{p}\right) d p,
\end{aligned}
$$

where

$$
\hat{\gamma}(\tau)=\gamma(t(\tau))
$$

Let

$$
E(\tau, x)=\exp \left[-\int_{a}^{x} \frac{b(y)}{\hat{\gamma}(\tau+\log (y / x)) \cdot y} d y\right] .
$$


A straightforward computation shows that

$$
\frac{\partial E}{\partial \tau}+x \frac{\partial E}{\partial x}=-\frac{b(x)}{\hat{\gamma}(\tau)} E(\tau, x) .
$$

Let $m(\tau, x)$ be given by

$$
m(\tau, x)=\frac{x u(\tau, x)}{E(\tau, x)},
$$

and let

$$
k(\tau, x, p)=2 d(p) \frac{b(x / p)}{\hat{\gamma}(\tau)} \frac{E(\tau, x / p)}{E(\tau, x)} .
$$

Substitution of (6.8) in (6.4) yields

$$
\frac{\partial m}{\partial \tau}+x \frac{\partial m}{\partial x}=\int_{\frac{1}{2}-\Delta}^{1}+\Delta k(\tau, x, p) m\left(\tau, \frac{x}{p}\right) d p,
$$

and $m$ must satisfy the initial condition

$$
m(0, x)=\phi(x)=\frac{x n_{0}(x)}{E(0, x)}
$$

and the boundary condition

$$
m(\tau, \alpha)=0
$$

The initial function $\phi$ given by (6.11) is an $L_{1}$-function if we assume $\left(\mathrm{H}_{S_{0}, n_{0}}\right) \frac{n_{0}(x)}{E(0, x)}$ is integrable on $[\alpha, 1]$.

Nota bene that $E(0, x)$ depends on $S_{0}$ and $w=W\left[n_{0}\right]$. This assumption is the analogue of assumption $\left(\mathrm{H}_{n_{0}}\right)$ mentioned in Section 2 .

So, for a fixed initial pair $S_{0}, n_{0}$, the nonlinear problem stated in Section 1 can be reduced to the linear (nonautonomous) problem given by (6.10)-(6.12). We call $m(\tau, x)$ a solution if $m$ is differentiable along the characteristics of Equation (6.10) (see Remark 4.1) and obeys (6.10), (6.11), and (6.12). We shall prove in this section that for all $\phi \in L_{1}[\alpha, 1]$ there does exist a unique solution of the initial-value problem (6.10)-(6.12), which we write abstractly as

$$
\frac{d m}{d \tau}=B m+C(\tau) m, \quad m(0)=\phi,
$$


where the unbounded closed operator $B$ is given by

$$
(B \psi)(x)=-x \frac{d \psi}{d x},
$$

having a domain

$$
\mathrm{D}(B)=\left\{\psi \in L_{1} \mid \psi \text { is absolutely continuous and } \psi(\alpha)=0\right\},
$$

and $C(\tau), \tau \geqslant 0$, defines a family of bounded operators on $L_{1}[\alpha, 1]$ :

$$
(C(\tau) \psi)(x)=\int_{\frac{1}{2}-\Delta}^{\frac{1}{2}+\Delta} k(\tau, x, p) m\left(\tau, \frac{x}{p}\right) d p .
$$

In Section 4 we have seen that $B$ generates a strongly continuous semigroup $e^{t B}$ given by

$$
\left(e^{t B} \psi\right)(x)=\psi\left(x e^{-t}\right) .
$$

Now a result of Kato yields that $B+C(\tau)$ "generates" a unique evolution operator (or solution operator) $V(\tau, \sigma)$. (See [14, Theorem 4.5].) This means that the solution of $(6.10)-(6.12)$ is given by

$$
m(\tau, \cdot ; \phi)=V(\tau, 0) \phi .
$$

Nota bene that the family $V(\tau, \sigma)$ depends on $w$; occasionally we shall write $V(\tau, \sigma ; w)$ if this dependence is to be emphasized.

The solution of the nonlinear equation (1.3) can be found in the following way. Let $S_{0}, n_{0}$ satisfy $\left(\mathrm{H}_{S_{0}, n_{0}}\right), w=W\left[n_{0}\right]$, and let $\phi$ be given by (6.11). Then the solution $n\left(t, x ; S_{0}, n_{0}\right)$ of the nonlinear problem (1.3)-(1.7) is given by

$$
n\left(t, x ; S_{0}, n_{0}\right)=e^{-D t} \frac{E(\tau(t), x)}{x}(V(\tau(t), 0 ; w) \phi)(x) .
$$

Notice carefully that $\tau=\tau(t)$ depends on $w=W\left[n_{0}\right]$. Now we have proved THEOREM 6.1

Let the initial pair $S_{0}, n_{0}$ satisfy condition $\left(\mathrm{H}_{S_{0}, n_{0}}\right)$. Then the nonlinear initial-value problem (1.3)-(1.7) has a unique solution $n=n\left(t, x ; S_{0}, n_{0}\right)$, $S=S\left(t ; S_{0}, w\right)$ where $w=W\left[n_{0}\right]$.

Remark 6.1. As we did in Section 4, we can represent the solution as a series, by applying a variation-of-constants formula to (6.13). (See the Appendix, proof of Theorem 8.4.) 


\section{THE EXISTENCE OF A GLOBALLY STABLE EQUILIBRIUM}

We ended Section 5 with the question whether there exists a (globally) stable size distribution. In this section we shall answer this question affirmatively. First, suppose that condition $\left(\mathrm{H}_{e}\right)$ of Section 5 is not satisfied. Then

$$
\lim _{t \rightarrow \infty} W\left(t ; S_{0}, w\right)=0 \quad \text { for all } S_{0}>0, \quad w>0 .
$$

From this we obtain

$$
\begin{aligned}
\left\|n\left(t ; S_{0}, n_{0}\right)\right\| & =\int_{\alpha}^{1} n\left(t, x ; S_{0}, n_{0}\right) d x \leqslant \frac{1}{\alpha} \int_{\alpha}^{1} x n\left(t, x ; S_{0}, n_{0}\right) d x \\
& =\frac{1}{\alpha} W\left(t ; S_{0}, w\right),
\end{aligned}
$$

yielding the following result:

THEOREM 7.1

If $\left(\mathrm{H}_{e}\right)$ is not satisfied, $S_{0}$ is an initial substrate concentration, and $n_{0}$ is an initial size distribution such that $\left(\mathrm{H}_{S_{1}, n_{0}}\right)$ is satisfied, then $\lim _{t \rightarrow \infty} n\left(t, \cdot ; S_{0}, n_{0}\right)=0$ in the $L_{1}$ sense.

During the rest of this section we assume that $\left(\mathrm{H}_{e}\right)$ is satisfied. Theorem 5.2 states

$$
\begin{aligned}
& \lim _{t \rightarrow \infty} S\left(t ; S_{0}, w\right)=S_{e}, \\
& \lim _{t \rightarrow \infty} W\left(t ; S_{0}, w\right)=W_{e},
\end{aligned}
$$

with $S_{e}$ and $W_{e}$ given by (5.7). Suppose that for all $t \geqslant 0$

$$
S\left(t ; S_{0}, w\right)=S_{e}, \quad W\left(t ; S_{0}, w\right)=W_{e},
$$

(this means that $S_{0}=S_{e}$ and $\left.w=W\left[n_{0}\right]=W_{e}\right)$; then $\gamma\left(t ; S_{0}, w\right)$ given by (6.1) is to be replaced by $\gamma\left(S_{e}\right)=D$. The solution $n\left(t, x ; S_{0}, n_{0}\right)$ can be found by applying the (linear) theory of Section 4, with $g(x)=\gamma\left(S_{e}\right) x=D x$, and $D(x)=D$. Theorem 3.7 states that in this case the dominant eigenvalue of the generator $A$ is 0 . Let us denote the corresponding eigenvector of $A$ by $\psi_{e}$, and let $\hat{n}_{e}$ be given by (3.15):

$$
\hat{n}_{e}(x)=\frac{E(x)}{D x} \psi_{e}(x)
$$

Corollary 4.6 states that

$$
n\left(t, \cdot ; S_{0}, n_{0}\right)=W_{e} \cdot \hat{n}_{e}(\cdot)+O\left(e^{-\epsilon t}\right), \quad t \rightarrow \infty,
$$


where $\hat{n}_{e}$ is normalized by the condition $W\left[\hat{n}_{e}\right]=1$. [See (4.11).] Let $n_{e}:=W_{e}$ $\cdot \hat{n}_{e} ;$ then

$$
W\left[n_{e}\right]=W_{e}
$$

We conclude that $n\left(t, \cdot ; S_{0}, n_{0}\right)$ approaches the equilibrium $n_{e}$ if $(\star)$ is satisfied. Theorem 5.2 states that $W\left(t ; S_{0}, w\right)$ and $S\left(t ; S_{0}, w\right)$ approach the equilibria $W_{e}$ and $S_{e}$ if $t$ tends to infinity. We can state our main result now.

\section{THEOREM 7.2}

Let $\left(\mathrm{H}_{e}\right)$ be satisfied, and let the initial pair $S_{0}, n_{0}$ satisfy condition $\left(\mathrm{H}_{S_{0}, n_{0}}\right)$. Then

$$
\lim _{t \rightarrow \infty} n\left(t, \cdot ; S_{0}, n_{0}\right)=n_{e}(\cdot) \quad \text { in the } L_{1} \text { sense. }
$$

This result is proved in the following section.

It is obvious that $\left(\mathrm{H}_{e}\right)$ is satisfied if and only if $D$ is below some critical value $D_{\mathrm{cr}}$. Theorems 7.1 and 7.2 have the following interpretation:

If $D<D_{\text {cr }}$, then a nontrivial steady state is reached.

If $D \geqslant D_{\text {cr }}$, then the population goes extinct.

\section{PROOF OF THE MAIN RESULT}

To prove Theorem 7.2 we shall make use of the theory of dynamical systems. We use the following notation. Let

$$
X=R \times L_{1}[\alpha, 1] .
$$

We denote an element of $X$ by $\langle S, n\rangle$. Then

$$
\|\langle S, n\rangle\|_{X}=|S|+\|n\|_{L_{1}}, \quad\langle S, n\rangle \in X
$$

defines a norm on $X$, and it is obvious that with this norm $X$ becomes a Banach space. Let the subset $Z$ of $X$ be given by

$$
Z=\left\{\left\langle S_{0}, n_{0}\right\rangle \in X \mid S_{0} \geqslant 0, n_{0} \in L_{1}^{+} \text {, and the pair } S_{0}, n_{0} \text { obeys }\left(\mathrm{H}_{S_{0}, n_{0}}\right)\right\} \text {. }
$$

If $\left\langle S_{0}, n_{0}\right\rangle \in Z$ and $w=W\left[n_{0}\right]$, then $\langle S(t), n(t)\rangle=\left\langle S\left(t ; S_{0}, w\right)\right.$, $\left.n\left(t ; S_{0}, n_{0}\right)\right\rangle$ is an element of $Z$, as one can see from Section 6 . Let $U(t): R^{+} \times Z \rightarrow Z$ be defined by

$$
U(t)\left\langle S_{0}, n_{0}\right\rangle=\langle S(t), n(t)\rangle .
$$


$U(t)$ is sometimes called a generalized dynamical system, where the adjective "generalized" takes account of the fact that $U(t)$ is only defined on a subset of $X$. For $\left\langle S_{0}, n_{0}\right\rangle \in Z$,

$$
\Gamma^{+}\left(\left\langle S_{0}, n_{0}\right\rangle\right)=\bigcup_{t \geqslant 0}\{\langle S(t), n(t)\rangle\}
$$

is called the orbit starting from $\left\langle S_{0}, n_{0}\right\rangle$. From Section 6 it is clear that $\Gamma^{+}\left(\left\langle S_{0}, n_{0}\right\rangle\right) \subset Z$ if $\left\langle S_{0}, n_{0}\right\rangle \in Z$. The following result follows immediately from Theorem 5.1 and the estimate (7.1).

LEMMA 8.1

For all $\left\langle S_{0}, n_{0}\right\rangle \in Z$, the orbit $\Gamma^{+}\left(\left\langle S_{0}, n_{0}\right\rangle\right)$ is bounded.

Boundedness of orbits is needed in order to prove precompactness. THEOREM 8.2

For all $\left\langle S_{0}, n_{0}\right\rangle \in Z$, the orbit $\Gamma^{+}\left(\left\langle S_{0}, n_{0}\right\rangle\right)$ is precompact.

The proof of this very important result is given in the Appendix.

The $\omega$-limit set $\Omega\left(\left\langle S_{0}, n_{0}\right\rangle\right)$ of the orbit starting from some $\left\langle S_{0}, n_{0}\right\rangle \in Z$ is the set of elements $\langle\Sigma, \nu\rangle \in X$ for which there exists a non-decreasing sequence $\left\{t_{n}\right\}, t_{n}>0, t_{n} \rightarrow \infty$ if $n \rightarrow \infty$, such that

$$
\left\|U\left(t_{n}\right)\left\langle S_{0}, n_{0}\right\rangle-\langle\Sigma, \nu\rangle\right\|_{X} \rightarrow 0 \quad \text { as } n \rightarrow \infty .
$$

\section{LEMMA 8.3}

Let $\left\langle S_{0}, n_{0}\right\rangle \in Z$. For all $\langle\Sigma, \nu\rangle \in \Omega\left(\left\langle S_{0}, n_{0}\right\rangle\right)$ we have $\Sigma=S_{e}$ and $W[\nu]=W_{e}$.

Proof. This follows immediately from Theorem 5.2.

The $\omega$-limit set is only of practical use if every element in it is contained in the domain of the generalized dynamical system.

LEMMA 8.4

If $\left\langle S_{0}, n_{0}\right\rangle \in Z$, then $\Omega\left(\left\langle S_{0}, n_{0}\right\rangle\right) \subset Z$.

Proof. Let $\left\langle S_{0}, n_{0}\right\rangle \in Z$ and $\langle\Sigma, \nu\rangle \in \Omega\left(\left\langle S_{0}, n_{0}\right\rangle\right)$. There exists a sequence $\left\{t_{k}\right\}$ satisfying $t_{k}>0, t_{k} \rightarrow \infty$ if $k \rightarrow \infty$, such that

$$
U\left(t_{k}\right)\left\langle S_{0}, n_{0}\right\rangle \rightarrow\langle\Sigma, \nu\rangle \quad \text { if } \quad k \rightarrow \infty .
$$

This yields $n\left(t_{k}, \cdot ; S_{0}, n_{0}\right) \rightarrow \nu, k \rightarrow \infty$ in the $L_{1}$ sense. Let $m(\tau, x)$ be given by

$$
E(\tau, x) m(\tau, x)=e^{D t} x n(t, x),
$$


where $\tau=\tau(t)=\int_{0}^{t} \gamma\left(S\left(t^{\prime} ; S_{0}, w\right)\right) d t^{\prime}, w=W\left[n_{0}\right]$, and $E(\tau, x)$ is given by (6.6). We denote by $t=t(\tau)$ the inverse function of $\tau=\tau(t)$. (See Section 6.) From the proof of Theorem 8.2 in the Appendix it follows that the set $\left\{e^{-D t(\tau)} m(\tau, \cdot) \mid \tau \geqslant 0\right\}$ is precompact in $L_{1}$. Hence there exists a subsequence $\left\{t_{k}^{\prime}\right\}$ of $\left\{t_{k}\right\}$ and an element $\phi \in L_{1}$ such that $e^{-D t_{k}^{\prime}} m\left(\tau_{k}^{\prime}, \cdot\right) \rightarrow \phi$ as $k \rightarrow \infty$. Here $\tau_{k}^{\prime}=\tau\left(t_{k}^{\prime}\right)$. The relation $(\star)$ yields

$$
e^{-D t_{k}^{\prime}} m\left(\tau_{k}^{\prime}, x\right) E\left(\tau_{k}^{\prime}, x\right)=x n\left(t_{k}^{\prime}, x\right) \text {. }
$$

If we let on both sides $k$ tend to infinity, we obtain $\phi(x) E(x)=x \nu(x)$, where

$$
E(x)=\exp \left(-\int_{\alpha}^{x} \frac{b(y)+D}{D y} d y\right)
$$

[We have used $\hat{\gamma}\left(\tau_{k}^{\prime}\right) \rightarrow \gamma\left(S_{e}\right)=D$ if $k \rightarrow \infty$.]

Lemma 8.1 states that $\Sigma=S_{e}$ and $W[\nu]=W_{e}$, and this yields that $\langle\Sigma, \nu\rangle$ obeys condition $\left(\mathrm{H}_{S_{0}, n_{0}}\right)$. As a consequence $\langle\Sigma, \nu\rangle \in Z$, which proves the result.

Although $U(t)$ does not define a dynamical system in the usual sense of the word $[U(t)$ only acts on a subset of $X]$, many results from dynamical-system theory remain valid.

\section{THEOREM 8.5}

For all $\left\langle S_{0}, n_{0}\right\rangle \in Z$, the $\omega$-limit set $\Omega\left(\left\langle S_{0}, n_{0}\right\rangle\right)$ is nonempty, compact, and invariant. Moreover, $U(t)\left\langle S_{0}, n_{0}\right\rangle \rightarrow \Omega\left(\left\langle S_{0}, n_{0}\right\rangle\right)$ as $t \rightarrow \infty$, with respect to the norm of $X$.

Proof. A straightforward computation, using Theorem 8.2 and Lemma 8.4 , shows that the proof of Theorem IV.4.1 of [22] can be carried over.

Now, let $\left\langle S_{0}, n_{0}\right\rangle \in Z$ and $\langle\Sigma, \nu\rangle \in \Omega\left(\left\langle S_{0}, n_{0}\right\rangle\right)$. (Theorem 8.4 assures that such an element $\langle\Sigma, \nu\rangle$ exists). Lemma 8.3 gives us

$$
S(t ; \Sigma, W[\nu])=S\left(t ; S_{e}, W_{e}\right)=S_{e},
$$

which means that an orbit starting from some element of $\Omega\left(\left\langle S_{0}, n_{0}\right\rangle\right)$ can be found by applying the linear theory of Section 4. Using $\gamma\left(S_{e}\right)=D$ (see Section 5), Theorem 3.7 states that the dominant eigenvalue satisfies $\lambda_{d}=0$ in this case. We obtain

$$
\left\|n(t ; \Sigma, \nu)-n_{e}\right\| \leqslant M e^{-\epsilon t}\|\nu\|
$$

for some constants $\epsilon, M>0$, where $n_{e}$ is normalized by (7.5). Here we have 
used Corollary 4.6. The invariance of $\Omega\left(\left\langle S_{0}, n_{0}\right\rangle\right)$ yields that for all $t \geqslant 0$ there exists a $\left\langle\Sigma^{-t}, \nu^{-t}\right\rangle \in \Omega\left(\left\langle S_{0}, n_{0}\right\rangle\right)$ such that $U(t)\left\langle\Sigma^{-t}, \nu^{-t}\right\rangle=\langle\Sigma, \nu\rangle$.
Hence

$$
\begin{aligned}
\left\|\langle\Sigma, \nu\rangle-\left\langle S_{e}, n_{e}\right\rangle\right\|_{X} & =\left\|U(t)\left\langle\Sigma^{-t}, \nu^{-t}\right\rangle-\left\langle S_{e}, n_{e}\right\rangle\right\|_{X} \\
& =\left\|n\left(t ; \Sigma^{-t}, \nu^{-t}\right)-n_{e}\right\|_{L_{1}} \\
& \leq M e^{-\epsilon t}\left\|\nu^{-t}\right\| \leqslant \frac{1}{\alpha} M e^{-\epsilon t} W\left[\nu^{-t}\right] \\
& =\frac{1}{\alpha} M W_{e} e^{-\epsilon t} .
\end{aligned}
$$

Here we have used $\Sigma^{-t}=S_{e}$, the inequality (8.5), and $W\left[\nu^{-t}\right]=W_{e}$. The inequality above holds for all $t \geqslant 0$, from which we conclude $\langle\Sigma, \nu\rangle=$ $\left\langle S_{e}, n_{e}\right\rangle$. We have proved the following result.

\section{THEOREM 8.6}

For all $\left\langle S_{0}, n_{0}\right\rangle \in Z$ we have $\Omega\left(\left\langle S_{0}, n_{0}\right\rangle\right)=\left\langle S_{e}, n_{e}\right\rangle$.

Combining this and Theorem 8.5 yields

$$
U(t)\left\langle S_{0}, n_{0}\right\rangle \rightarrow\left\langle S_{e}, n_{e}\right\rangle, \quad t \rightarrow \infty,
$$

and this proves Theorem 7.2.

\section{CONCLUDING REMARKS}

The continuous culture of microorganisms has become a technique of great importance in microbiology. However, up till now, most of the models describing continuous culture populations have not incorporated any structure distinguishing between the individuals of such a population. The paper of Gyllenberg [9] forms an exception. He assumes that organisms can be distinguished from each other according to their age. Another exception is formed by a paper of Diekmann et al. [7], which will be discussed below. In this paper we have considered a continuous culture of cells, whose individuals are assumed to be characterized by their size only. An important feature of our (nonlinear) model is its analytic solvability. This is due to several assumptions made in this paper. For instance, the resulting mathematical theory fails in both of the following cases:

(1) One does not restrict oneself to the case of exponential growth. (See Section 5.)

(2) One assumes that fission is into two equal parts. In this case there does not exist a stable size distribution for the associated linear problem if $g(x)=\gamma x$. (See $[6$, Section 8].) 
In this paper we have assumed that division can be described by a function $b(x)$, which quantity can be interpreted as the probability per unit of time for a cell with size $x$ to divide. In [7] Diekmann et al. present a second possibility for describing fission. They assume the existence of a function $p(x)$ describing the chance per unit of size that a cell will divide at size $x$, i.e., $\int_{x_{1}}^{x_{2}} p(x) d x$ is the fraction of cells dividing between $x_{1}$ and $x_{2}$. They call this the stochastic threshold model. The following relation between $g, b$, and $p$ can be deduced:

$$
b(x)=g(x) \frac{p(x)}{1-\int_{\alpha}^{x} p(\xi) d \xi},
$$

or equivalently,

$$
p(x)=\frac{b(x)}{g(x)} \exp \left(-\int_{a}^{x} \frac{b(\xi)}{g(\xi)} d \xi\right)
$$

[Compare this with condition $\left(\mathrm{H}_{b}\right)(4)$ of Section 1.] Both descriptions yield the same results if $g$ only depends on $x$. However, if (as in our case) $g$ depends through nutrient limitation (or any other environmental factor) on time,

$$
g=\gamma(S) g(x),
$$

then the nonlinearity $\gamma(S)$ only causes a deformation of the time axis if one works with the stochastic threshold model. (See [7].) In that case, a change of the dilution rate $D$ will only cause a multiplication of the total population size by some factor. In our case, a change of the dilution rate $D$ will also cause a deformation of the shape of the stable size distribution, and this provides an experimental test of the correctness of our model.

We intend to study more general nonlinear models, describing proliferating cell populations, in the near future.

\section{APPENDIX}

For the proof of Theorem 4.3 and Theorem 8.2 we need the following lemma.

\section{LEMMA}

Let $K \subset L_{1}[\alpha, 1]$ be bounded, and suppose that every $\phi \in K$ is absolutely continuous and satisfies $\int_{\alpha}^{1}\left|\phi^{\prime}(x)\right| d x \leqslant M$, where $M$ is a positive constant not depending on $\phi$. Then $K$ is precompact. 
Proof. We must proof that for all $\epsilon>0$ there exists a $\delta>0$ such that for all $\phi \in K$

$$
\int_{\alpha}^{1}|\phi(x+h)-\phi(x)| d x<\epsilon \quad \text { if } \quad|h|<\delta .
$$

Let $\phi \in K$ and $h>0$. Then

$$
|\phi(x+h)-\phi(x)| \leqslant \int_{x}^{x+h}\left|\phi^{\prime}(t)\right| d t .
$$

Hence

$$
\begin{aligned}
& \int_{\alpha}^{1}|\phi(x+h)-\phi(x)| d x \\
& \quad \leqslant \int_{\alpha}^{1}\left\{\int_{x}^{x+h}\left|\phi^{\prime}(t)\right| d t\right\} d x \\
& \quad=\int_{\alpha}^{\alpha+h}\left|\phi^{\prime}(t)\right|\left\{\int_{\alpha}^{t} d x\right\} d t+\int_{\alpha+h}^{1}\left|\phi^{\prime}(t)\right|\left\{\int_{t-h}^{t} d x\right\} d t \\
& \quad \leqslant h \int_{\alpha}^{1}\left|\phi^{\prime}(t)\right| d t \quad \\
& \quad=h M<\epsilon \quad \text { if } \quad h<\frac{\epsilon}{M} .
\end{aligned}
$$

A similar estimate can be found for negative $h$, and this proves the result.

Proof of Theorem 4.3 (for the case $g(x)=\gamma x$ ). The mapping $\phi \rightarrow m_{1}(t ; \phi)$ where $m_{1}$ is determined by (4.7)-(4.8) defines a family of bounded operators, which we denote by $S_{1}(t): m_{1}(t ; \phi)=S_{1}(t) \phi$. A straightforward computation, using (4.3), (4.4), (4.7), and (4.8), shows that

$$
\begin{aligned}
\left(S_{1}(t) \phi\right)(x) & =\int_{0}^{t}\left\{\int_{\frac{1}{2}-\Delta}^{\frac{1}{2}+\Delta} k\left(p, x e^{-\gamma \tau}\right) \cdot \phi\left(\frac{x}{p} e^{-\gamma t}\right) d p\right\} d \tau \\
& =\int_{\frac{1}{2}-\Delta}^{\frac{1}{2}+\Delta} \phi\left(\frac{x}{p} e^{-\gamma t}\right) \cdot\left\{\int_{0}^{t} k\left(p, x e^{-\gamma \tau}\right) d t\right\} d p
\end{aligned}
$$

where we have substituted $g(x)=\gamma x$. If $p$ is replaced by the new variable $z=(x / p) e^{-\gamma t}$, we obtain

$$
\left(S_{1}(t) \phi\right)(x)=x e^{-\gamma t} \int_{x e^{-\gamma t} /\left(\frac{1}{2}+\Delta\right)}^{x e^{-\gamma t} /\left(\frac{1}{2}-\Delta\right)} \frac{\phi(z)}{z^{2}}\left\{\int_{0}^{t} k\left(\frac{x}{z} e^{-\gamma t}, x e^{-\gamma \tau}\right) d \tau\right\} d z
$$

It is clear that $S_{1}(t) \phi$ is absolutely continuous. Let $\left(L_{1}(t) \phi\right)(x)=$ 
$(d / d x)\left(S_{1}(t) \phi\right)(x)$. It follows directly that

$$
\begin{aligned}
\left(L_{1}(t) \phi\right)(x)= & \frac{1}{x}\left(S_{1}(t) \phi\right)(x)+x e^{-\gamma t} \int_{x e^{-\gamma t} /\left(\frac{1}{2}+\Delta\right)}^{x e^{-\gamma t} /\left(\frac{1}{2}-\Delta\right)} \frac{\phi(z)}{z^{2}} \\
& \times\left\{\int_{0}^{t} \frac{d}{d x}\left[k\left(\frac{x}{z} e^{-\gamma t}, x e^{-\gamma \tau}\right)\right] d \tau\right\} d z
\end{aligned}
$$

where we have used that $k\left(\frac{1}{2}-\Delta, \cdot\right)=k\left(\frac{1}{2}+\Delta, \cdot\right)=0$. Using (2.8), we find

$$
\begin{aligned}
\frac{d}{d x}\left\{k\left(\frac{x}{z} e^{-\gamma t}, x e^{-\gamma \tau}\right)\right\}= & \frac{4}{z} e^{-\gamma t} d^{\prime}\left(\frac{x}{z} e^{-\gamma t}\right) \frac{b\left(z e^{\gamma(t-\tau)}\right) E\left(z e^{\gamma(t-\tau)}\right)}{E\left(x e^{-\gamma \tau}\right)} \\
& +4 d\left(\frac{x}{z}\right) e^{-\gamma t} \frac{(b E)\left(z e^{\gamma(t-\tau)}\right)}{E\left(x e^{-\gamma \tau}\right)} \\
& \cdot \frac{b\left(x e^{-\gamma \tau}\right)+D\left(x e^{-\gamma \tau}\right)}{\gamma x} .
\end{aligned}
$$

Observe that $z e^{\gamma(t-\tau)} \leqslant 1$ implies that $x e^{-\gamma \tau} \leqslant \frac{1}{2}+\Delta=\beta$, because $z=$ $(x / p) e^{-\gamma t}$ for some $p \in\left[\frac{1}{2}-\Delta, \frac{1}{2}+\Delta\right]$. Let

$$
\begin{aligned}
& D_{\max }:=\max \{D(x) \mid x \in[\alpha, \beta]\}, \\
& d_{\max }:=\max \left\{d(p) \mid p \in\left[\frac{1}{2}-\Delta, \frac{1}{2}+\Delta\right]\right\}, \\
& d_{\max }^{\prime}:=\max \left\{\mid d^{\prime}(p) \| p \in\left[\frac{1}{2}-\Delta, \frac{1}{2}+\Delta\right]\right\} .
\end{aligned}
$$

Notice that $d_{\max }^{\prime}$ is well defined because of hypothesis $\left(\mathrm{H}_{d}\right)$. We obtain the following estimate:

$$
\begin{aligned}
\left|\frac{d}{d x} k\left(\frac{x}{z} e^{-\gamma t}, x e^{-\gamma \tau}\right)\right| \leqslant & \frac{(4 / \alpha) e^{-\gamma t} d_{\max }^{\prime}}{E(\beta)} \cdot b\left(z e^{\gamma(t-\tau)}\right) \cdot E\left(z e^{\gamma(t-\tau)}\right) \\
& +\frac{4 d_{\max }}{E(\beta)} \cdot \frac{b(\beta)+D_{\max }}{\gamma \alpha} \cdot b\left(z e^{\gamma(t-\tau)}\right) E\left(z e^{\gamma(t-\tau)}\right) \\
:= & C \cdot b\left(z e^{\gamma(t-\tau)}\right) E\left(z e^{\gamma(t-\tau)}\right) .
\end{aligned}
$$

We deduce

$$
\begin{aligned}
\left|\left(L_{1}(t) \phi\right)(x)\right| \leqslant & \frac{1}{\alpha}\left|\left(S_{1}(t) \phi\right)(x)\right|+C e^{-\gamma t} \int_{x e^{-\gamma t} /\left(\frac{1}{2}+\Delta\right)}^{x e^{-\gamma t} /\left(\frac{1}{2}-\Delta\right)} \frac{|\phi(z)|}{z^{2}} \\
& \times\left\{\int_{0}^{t} b\left(z e^{\gamma \tau}\right) E\left(z e^{\gamma \tau}\right) d \tau\right\} d z .
\end{aligned}
$$


Using

$$
\int_{0}^{t} b\left(z e^{\gamma \tau}\right) E\left(z e^{\gamma \tau}\right) d \tau=\int_{z}^{z e^{\gamma t}} \frac{b(\xi) E(\xi)}{\gamma \xi} d \xi \leqslant 1,
$$

we obtain

$$
\left|\left(L_{1}(t) \phi\right)(x)\right| \leqslant \frac{1}{\alpha}\left|\left(S_{1}(t) \phi\right)(x)\right|+\frac{C e^{-\gamma t}}{\alpha^{2}}\|\phi\| .
$$

Consequently

$$
\left\|L_{1}(t)\right\| \leqslant \frac{1}{\alpha}\left\|S_{1}(t)\right\|+\frac{C e^{-\gamma t}}{\alpha^{2}} .
$$

This result and the former lemma yield the compactness of $S_{1}(t)$. Let $S_{i}(t)$ be defined by the relation

$$
S_{i}(t) \phi=m_{i}(t ; \phi) \text {. }
$$

Using the recurrence relation (4.7)-(4.8) and the fact that the integral expression in (4.8) is a standard Riemann integral, we find that $S_{i}(t)$ is compact for all $i \geqslant 1$, and this result holds for all $t \geqslant 0$. Now, the proof is completed by the observation

$$
T(t)=\sum_{i=1}^{\infty} S_{i}(t) \quad \text { if } \quad t \geqslant G(1)
$$

Proof of Theorem 8.2. Let $Z_{1}$ be the subset of $Z$ containing all elements $\left\langle S_{0}, n_{0}\right\rangle$ satisfying

$$
S\left(t ; S_{0}, w\right) \geqslant \frac{1}{2} S_{e}, \quad t \geqslant 0,
$$

where $w=W\left[n_{0}\right]$. By definition, $U(t) Z_{1} \subset Z_{1}, t \geqslant 0$. Because of Theorem 5.2 , every orbit $\Gamma^{+}\left(\left\langle S_{0}, n_{0}\right\rangle\right)$ enters $Z_{1}$ for $t$ large enough, and for that reason we may restrict ourselves to initial pairs $\left\langle S_{0}, n_{0}\right\rangle$ which are elements of $Z_{1}$.

The solution operator $V(\tau, \sigma)$, which has been defined in Section 6 , can be represented as a series:

$$
V(\tau, \sigma)=\sum_{i=0}^{\infty} V_{i}(\tau, \sigma)
$$


and the elements of this series can be computed from the following recurrence relation:

$$
\begin{aligned}
V_{0}(\tau, \sigma) & =e^{(\tau-\sigma) B}, \\
V_{i+1}(\tau, \sigma) & =\int_{\sigma}^{\tau} e^{\left(\tau-\tau^{\prime}\right) B} C\left(\tau^{\prime}\right) V_{i}\left(\tau^{\prime}, \sigma\right) d \tau^{\prime} .
\end{aligned}
$$

From this, it is clear that also $U(t)$ can be written as a series:

$$
U(t)=\sum_{i=0}^{\infty} U_{i}(t),
$$

where

$$
\begin{aligned}
& U_{0}(t)\left\langle S_{0}, n_{0}\right\rangle=\left\langle S\left(t ; S_{0}, w\right), n^{0}\left(t ; S_{0}, n_{0}\right)\right\rangle \\
& U_{i}(t)\left\langle S_{0}, n_{0}\right\rangle=\left\langle 0, n^{i}\left(t ; S_{0}, n_{0}\right)\right\rangle, \quad i \geqslant 1,
\end{aligned}
$$

where $n^{i}\left(t, x ; S_{0}, n_{0}\right)$ can be determined from (6.15):

$$
n^{i}\left(t, x ; S_{0}, n_{0}\right)=e^{-D t} \frac{E(\tau(t), x)}{x}\left(V_{i}(\tau(t), 0 ; w) \phi\right)(x),
$$

where $\phi$ is given by (6.11). Now

$$
V_{0}(\tau, 0)=0 \quad \text { if } \quad \tau \geqslant \log \frac{1}{\alpha},
$$

because

$$
\begin{gathered}
\left(V_{0}(\tau, 0) \phi\right)(x)=\phi\left(x e^{-\tau}\right) \\
\tau(t)=\int_{0}^{t} \gamma\left(t^{\prime}\right) d t^{\prime}=\int_{0}^{t} \gamma\left(S\left(t^{\prime} ; S_{0}, w\right)\right) d t^{\prime} \\
\geqslant \int_{0}^{t} \gamma\left(\frac{1}{2} S_{e}\right) d t^{\prime}=\gamma\left(\frac{1}{2} S_{e}\right) t \geqslant \log \frac{1}{\alpha}
\end{gathered}
$$

if

$$
t \geqslant t_{1} \stackrel{\text { def }}{=} \frac{1}{\gamma\left(\frac{1}{2} S_{e}\right)} \log \frac{1}{\alpha}
$$

Now we can prove the following result.

Let $t \geqslant t_{1}$, and $K$ be a bounded subset of $Z_{1}$. Then $U(t) K$ is precompact. 
Proof. If $t \geqslant t_{1}$, then $U_{0}(t)\left\langle S_{0}, n_{0}\right\rangle=\left\langle S\left(t ; S_{0}, w\right), 0\right\rangle$, which, together with Theorem 5.1, yields that $U_{0}(t)$ is compact with respect to $Z_{1}$ for $t \geqslant t_{1}$. In a way which is very similar to the proof of Theorem 4.3 , it can be shown that for $i \geqslant 1, U_{i}(t)$ is compact with respect to $Z_{1}$, for all $t \geqslant 0$. The proof, which is slightly more difficult, uses the fact $S\left(t ; S_{0}, w\right)$ [and therefore the individual growth $\left.\gamma\left(t ; S_{0}, w\right) x\right]$ is bounded from above and below for all $t \geqslant 0$, uniformly in $\left\langle S_{0}, n_{0}\right\rangle \in K$.

Now $\Gamma^{+}\left(\left\langle S_{0}, n_{0}\right\rangle\right)=\left\{U(t)\left\langle S_{0}, n_{0}\right\rangle \mid t \leqslant t_{1}\right\} \cup U\left(t_{1}\right)\left\{U(s)\left\langle S_{0}, n_{0}\right\rangle \mid s \geqslant 0\right\}$, and Theorem 8.1 and the above lemma yield that $\Gamma^{+}\left(\left\langle S_{0}, n_{0}\right\rangle\right)$ is precompact.

I would like to thank Odo Diekmann for some valuable discussions on the subject.

\section{REFERENCES}

1 G. I. Bell and E. C. Anderson, Cell growth and division. I. A mathematical model with applications to cell volume distributions in mammalian suspension cultures, Biophys. J. 7:329-351 (1967).

2 E. C. Anderson and D. F. Petersen, Cell growth and division. II. Experimental studies of cell volume distributions in mammalian suspension cultures, Biophys. J. 7:353-364 (1967).

3 G. I. Bell, Cell growth and division. III. Conditions for balanced exponential growth in a mathematical model, Biophys. J. 8:431-444 (1968).

4 E. C. Anderson, G. I. Bell, D. F. Petersen, and R. A. Tobey, Cell growth and division. IV. Determination of volume growth rate and division probability, Biophys. $J$. 9:246-263 (1969).

5 O. Diekmann, The dynamics of structured populations: Some examples, in Proceedings of the International Conference on Mathematics in Biologv and Medicine, Bari, Italy, to appear.

6 O. Diekmann, H. J. A. M. Heijmans, and H. R. Thieme, On the stability of the cell size distribution, J. Math. Biol., 19:227-248 (1984).

7 O. Diekmann, H. A. Lauwerier, T. A. Aldenberg, and J. A. J. Metz, Growth, fission and the stable size distribution, J. Math. Biol. 18:135-148 (1983).

8 A. G. Fredrickson, D. Ramkrishna, and H. M. Tsuchiya, Statistics and dynamics of procaryotic cell populations, Math. Biosci. 1:327-374 (1967).

9 M. Gyllenberg, Nonlinear age-dependent population dynamics in continuously propagated bacterial cultures, Math. Biosci. 62:45-74 (1982).

10 J. K. Hale, Theory of Functional Differential Equations, Springer, 1977.

11 H. J. A. M. Heijmans, An eigenvalue problem related to cell growth, J. Math. Anal. Appl., to appear.

12 D. Herbert, R. Elsworth, and R. C. Telling, The continuous culture of bacteria, a theoretical and experimental study, J. Gen. Microbiol. 14:601-622 (1956).

13 S. B. Hsu, S. Hubbell, and P. Waltman, A mathematical theory for single-nutrient competition in continuous cultures of micro-organisms, SIAM J. Appl. Math. $32: 366-383$ (1977). 
14 T. Kato, Linear evolution equations of "hyperbolic" type, J. Fac. Sci. Univ. Tokyo Sect. I 17:241-258 (1970).

15 A. L. Koch and M. Schaechter, A model for statistics of the cell division process, $J$. Gen. Microbiol. 29:435-454 (1962).

16 P. R. Painter and A. G. Marr, Mathematics of microbial populations, Annual Rev. Microbiol. 22:519-548 (1968).

17 A. Pazy, Semigroups of Linear Operators and Applications to Partial Differential Equations, Springer, New York, 1983.

18 I. Sawashima, On spectral properties of some positive operators, Natur. Sci. Dept. Ochanomizu Univ. 15:53-64 (1964).

19 H. H. Schaefer, Banach Lattices and Positive Operators, Springer, 1974.

$20 \mathrm{~J}$. W. Sinko and W. Streifer, A model for populations reproducing by fission, Ecology 48:910-918 (1967).

21 W. J. Voorn, Statistics of cell size in the steady state, Ph.D. Thesis, Univ. of Amsterdam, 1983.

22 J. A. Walker, Dynamical Systems and Evolution Equations, Plenum, New York, 1980. 